\title{
Effect on weight gain of routinely giving albendazole to preschool children during child health days in Uganda: cluster randomised controlled trial
}

In 2007 a letter from the Cochrane Collaboration to the $B M J$ pointed out that the authors' analysis of the primary endpoint, weight gain, did not take account of the cluster randomised design (BMJ 2006;333:122, doi:10.1136/bmj.38877.393530. 7C). The Cochrane reviewers subsequently posted a rapid response detailing the correction they considered necessary

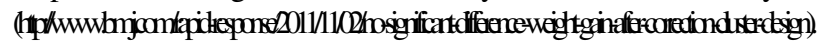
The paper should have been corrected at that time but the $B M J$ did not pursue negotiations over the wording and the correction was not published (http://www.bmj.com/content/333/7559/122/rr/618670). The authors would now like to make the following correction.

In our paper, we inadvertently failed to adjust the descriptive statistics for weight gain to take account of the study's cluster randomised design. The primary analyses of the study, the multivariate regressions, were, however, adjusted for cluster based sampling. The results section of the abstract in the published paper is based on the multivariate analysis and does not require correction.

The second paragraph of the results section, which in the published paper reads "Table 2 shows that at least two measurements of body weight were made on 14940 treated children and 13055 control children, and that there was a statistically significant difference in extra weight gained of 154 $\mathrm{g}(95 \%$ confidence interval 96 to $214, \mathrm{P}<0.01)$. This is equivalent to an extra $166 \mathrm{~g}$ per year ( 16 to $316 \mathrm{~g}$ ) or nearly $10 \%$ of average initial body weight," should have read:

"Table 2 shows that at least two measurements of body weight were made on 14940 treated children and 13055 control children. When adjusted for cluster randomisation, there was no statistically significant difference in total extra weight gained: mean weight gain in the treatment group $2413 \mathrm{~g}(95 \%$ confidence interval 2295 to 2533 ) and in the control group 2259 $\mathrm{g}$ (2121 to 2396); difference in weight gained $154 \mathrm{~g} \mathrm{(-19.7} \mathrm{to}$ $330 \mathrm{~g})$."

We are in discussion with the $B M J$ about the need for any further clarification about the analysis and reporting of our data.

Cite this as: BMJ 2012;345:e8724

(๑) BMJ Publishing Group Ltd 2012 